\title{
Endodermal progenitor cells isolated from mouse pancreas
}

\author{
Lisa Samuelson ${ }^{1}$, Natasha Wright ${ }^{1}$, David Allen Gerber ${ }^{1,2^{*}}$ \\ ${ }^{1}$ Department of Surgery, University of North Carolina, Chapel Hill, USA; \\ ${ }^{2}$ Lineberger Cancer Center, University of North Carolina, Chapel Hill, USA; \\ ${ }^{*}$ Corresponding Author: david_gerber@med.unc.edu
}

Received 11 June 2011; revised 23 July 2011; accepted 5 August 2011.

\begin{abstract}
In this study we have isolated and identified a progenitor cell population located in murine pancreatic tissue. This cell population has specific properties that make it different from previously described candidate pancreatic progenytor cell populations. It is located in the periductal region outside the islet architecture yet the cells have features consistent with endodermal development and endocrine functions within the islet. Cells are proliferative and capable of differentiation into pancreatic lineages expressing pancreatic associated transcription factors and proteins and maintaining the ability to produce insulin.
\end{abstract}

Keywords: Pancreatic Progenitor; Diabetes; Sca-1; Murine Pancreas

\section{INTRODUCTION}

Diabetes has reached epidemic proportions in the U.S. with an estimated prevalence of $6.3 \%$ of the U.S. population (translating into more than 18 million people) currently diagnosed with the disease [1]. Diabetes affected an estimated 171 million people worldwide in 2000 , and this number is predicted to rise to 366 million by 2030 , owing to increases in age, obesity, and urbanization of the world's population [2]. This rising incidence of patients diagnosed with diabetes drives the qu est for the development of novel therapies.

Diabetes is unique in that there is already a preexisting protocol of transplantation of donor beta cells to diabetic recipients which ameliorates the disease. However, the requirement of large numbers of beta cells and a shortage of donor tissue limits the procedure to select cases. The need for a highly proliferative and easily accessible cell population that can replace transplanted islets has led to the search for stem cell populations that can be used to treat the disease. Numerous investigators have attempted to identify and isolate a pancreatic stem or progenitor cell using distinct markers [3-7]. While a great deal of information is known about pancreatic development from the embryonic period through adulthood, there have been tremendous challenges associated with characterizing a putative pancreatic or islet stem cell. While the presence of precursor cells has been debated over the past decade [8-10], the potential presence of a precursor cell remains a possibility, as demonstrated by Liu et al via cell tracing experiments [11]. One group has established a murine pancreatic stem cell line that expresses PDX-1 but does not express select transcription factors such as Ngn3, BETA2/NeuroD, Pax4, Pax6, Isl-1, Nkx2.2 or Nkx6.1 [12]. Populations in mouse and human have been derived from pancreatic ductal cell components and maintained in long-term culture, where they could differentiate into multi-lineage cell types [13-15]. More recent experiments have mechanistically demonstrated how pancreatic-duct cells can serve as a source of regeneration [16]. The challenge with many of these populations was that they were characterized retrospectively after isolation. Petropavlovskaia et al. characterized a "small cell" isolated from islets that demonstrates expression of several pancreatic markers (insulin, glucagon, somatostatin, pancreatic polypeptide, etc.) during culture but doesn't express some of the stem cell related markers (e.g. c-kit, CD34) [17]. Additionally the identified cells demonstrate a very low level of proliferation.

Other researchers have studied embryonic stem cells to explore if they could be driven towards a pancreatic phenotype $[18,19]$. Some researchers have induced embryonic stem cells to a pancreatic precursor cell based on the cell's expression of Insulin I, Insulin II, PDX1 and other early marker proteins such as peptide YY (PYY) and glucagon [20,21]. Some investigators have focused on the expression of $\mathrm{Ngn} 3$ as a marker for a pancreatic progenitor cell during embryogenesis [22]. Select studies have provided direct evidence that Ngn3+ cells are potential islet progenitors during embryogenesis as well as 
in adult mice, and that $\mathrm{Pdx} 1^{+}$cells give rise to all three types of pancreatic tissue: exocrine, endocrine and ductal [22]. Ngn3 is required for specification of pancreatic endocrine cells along with additional molecules, such as Notch family members $[23,24]$. Ngn3 + cells are unipotent at the single-cell level but together as a population they are multipotent [25]. Other researchers have focused on fetal pancreatic duct cells as the origin of a multipotential cell population capable of differentiating into insulin-producing cells [26]. In addition there have been studies looking at clonal identification of multipotent precursors from adult mouse pancreas $[27,28]$. Under further selection, these cells have produced distinct populations of pancreatic $\beta$-, $\alpha$ - and $\delta$-cells, pancreatic exocrine and stellate cells, and neuronal and glial cells $[27,28]$. Additional investigators have looked at non- $\beta$ cell populations (e.g. $\alpha$-cells, gland cells, etc.) as a source of potential beta cells $[29,30]$.

We have identified a pancreatic progenitor cell isolated from mouse pancreas. This highly proliferative cell population is purified based on expression of stem cell antigen 1 (Sca-1). Cells express many transcription factors associated with pancreatic development and also have the ability to produce proteins associated with pancreatic endocrine function. These cells also that the ability to secret a basal amount of insulin.

\section{MATERIALS AND METHODS}

\subsection{Reagents}

Chemicals were obtained from the Sigma Chemical Company (St. Louis, MO) unless otherwise stated. Sca-1 antibody was purchased from BD Biosciences (San Jose, CA).

\subsection{Mice}

C57BL/6 mice breeding pairs were purchased from the Jackson Laboratory (Bar Harbor, ME). All animals were maintained on a rodent chow under a constant day/night cycle. 2 - 3 week old mice were used in all experiments. All care and use of animals was approved by the Institutional Animal Care and Use Committee at the University of North Carolina at Chapel Hill in accordance with the principles and procedures outlined in the National Institutes of Health Guide for the Care and Use of Laboratory Animals.

\subsection{Enrichment, Cell Sorting, and Culture}

Pancreatic tissue from 2 week old C57Bl/6 mice was removed and the tissue was digested with Liberase Blendzyme 3 (Roche) in a $36^{\circ}$ water bath for 15 min with agitation every 5 minutes. The tissue was then sheared with a $22 \mathrm{G}$ needle and tweezers and pipetted until all cells were disassociated. Cell sorting was performed using magnetic activated cell sorting (MACS ${ }^{\circledR}$, Miltenyi, Biotec, Inc.). The cells were separated using a Sca-1 antibody conjugated to mini-magnetic beads (Miltenyi Biotec Inc.; Auburn, CA, (http://www.milten yibiotec.com) according to the manufacturer's instructions. $\mathrm{Scal}^{+}$cells were eluted with a purity of $>94 \%$ by flow cytometry and $>80 \%$ viability. Standard culture conditions involved plating the cells on tissue culture dishes coated with Fibronectin and Concanavalin A in $2 \mathrm{ml}$ of DMEM (Dulbecco's Modified Eagle's medium) and 10\% FBS suPplemented with $10 \mathrm{ng} / \mathrm{ml}$ BMP-4 (R \& D Systems), 1400 U/ml ESGRO(Millipore, Temecula, CA), and B27 supplement (Invitrogen, Carlsbad, CA). Cellular density was $1 \times 10^{6}$ cells per $35 \mathrm{~mm}$ well. The cells were cultured in a $5 \% \mathrm{CO}_{2} / 95 \%$ room air incubator at $37^{\circ} \mathrm{C}$. The cells were passaged when they achieved $>75 \%$ confluence on the tissue culture dishes.

\subsection{Microscopy}

The cells in culture were visualized with either a Zeiss Axiovert 100 inverted fluorescent microscope and images were captured with Zeiss Axiovision 3.1 software or an Olympus BX61 Upright Fluorescence with Improvision's Velocity software provided by the Microscopy Services lab in the department o Molecular and Cellular Pathology.

\subsection{Fluorescent Immunophenotyping and Flow Cytometry}

Cells were stained for immunofluorescense using antibodies directly labeled with the relevant fluoroprobe. Cells were considered positive when fluorescence was greater than $95 \%$ of the negative control cells. Isotype control antibodies were used as negative controls. Cells were suspended in room temperature PBS containing 2\% FBS at $1 \times 10^{6}$ cells per ml. Antibodies were primary conjugates against Sca-1 (BD Biosciences; San Jose, CA). Cells were incubated for 20 minutes, centrifuged and resuspended in cold buffer at $1 \times 10^{6}$ per ml.

\subsection{Histology and Immunohistochemical Characterization}

Immunohistochemistry was performed by an indirect immunoperoxidase procedure for localization of select proteins (Monga et al., 2001). Primary antibody was against Sca-1 (BD Biosciences; San Jose, CA). The secondary antibody was from Vector Laboratories (Burlingame, $\mathrm{CA}$ ) and the signal was detected using the $\mathrm{ABC}$ Elite and DAB kits (Vector Laboratories, Burlingame, $\mathrm{CA}$ ). DAB staining was toned with DAB Enhancer (Vector Laboratories) and counterstained with hemotoxylin. Slides were 
blocked with Protein Blocking Agent (Thermo Electron Solutions; Pittsburgh, PA). For negative control, sections were incubated with isotype control antibodies. All stained slides were viewed on the Nikon MicrophotFXA microscope. It is equipped with an Optronics DEI 750 3-chip CCD camera and a Q Imaging Micropublisher CCD camera for digital image acquisition. Images were captured on an Apple Power Macintosh G3 computer utilizing Q Imaging software and saved using Adobe Photoshop CS2 software.

\subsection{RT-PCR}

Polymerase chain reaction (PCR) analysis was performed on total RNA extracted from isolated Sca-1 positive and negative cells. A total of $2 \mu \mathrm{g}$ of total RNA was used as a template to create complementary DNA as per the manufacturer's instructions for the Retroscript kit from (Ambion; Austin, TX). The sequences for selected primers are listed in Supplemental Data Table 1. The generated complementary DNA was then subjected to a denaturing temperature of $95^{\circ} \mathrm{C}$ for 2 minutes, followed by further denaturation at $95^{\circ} \mathrm{C}$ for $1 \mathrm{~min}$, annealing temperatures of $55^{\circ} \mathrm{C}-70^{\circ} \mathrm{C}$ for 45 seconds, and extension for 60 seconds at $72^{\circ} \mathrm{C}$ for 50 cycles, followed by a final extension at $72^{\circ} \mathrm{C}$ for 5 minutes.

Table 1. RT- PCR primers.

\begin{tabular}{|c|c|c|c|}
\hline Primer & 5'-3' & Annealing Temp & Size \\
\hline Ptf1alpha & $\begin{array}{l}\text { catagagaacgaaccaccctttgag } \\
\text { cttgagacaggtcctttgaggcacg }\end{array}$ & 60 & 294 \\
\hline Nkx2.2 & $\begin{array}{l}\text { aaaggtatggaggtgacgect } \\
\text { tcatgttgcgggtcatgtcga }\end{array}$ & 54 & 190 \\
\hline Ngn3 & $\begin{array}{l}\text { aagagcgagttggcactgagc } \\
\text { gcgtatcgcctggtgtcgaa }\end{array}$ & 56 & 223 \\
\hline Insulin 1 & $\begin{array}{l}\text { tagtgaccagctataatcagagac } \\
\text { agccaaggtctgaaggtc }\end{array}$ & 70 & 288 \\
\hline Insulin 2 & $\begin{array}{l}\text { ccctgctggecetgctctt } \\
\text { aggtctgaaggtcacctgct }\end{array}$ & 60 & 213 \\
\hline Nestin & $\begin{array}{l}\text { ggagagtcgcttagaggtgc } \\
\text { gaagagaaccgaaaggactg }\end{array}$ & 58 & 327 \\
\hline Glut 2 & $\begin{array}{l}\text { ggataaattcgcctggatga } \\
\text { tcaaggtcttggtttcctt }\end{array}$ & 53 & 298 \\
\hline NeuroD & $\begin{array}{l}\text { cttggccaagaactacatctgg } \\
\text { ccgttgaagagaaagtttgtgc }\end{array}$ & 53 & 430 \\
\hline CK19 & $\begin{array}{l}\text { ctgcagatgacttcagaacc } \\
\text { ggccatgatctcatactgac }\end{array}$ & 62 & 299 \\
\hline Pax 6 & $\begin{array}{l}\text { tcacagcggagtgaatcag } \\
\text { cccaagcaaagatggaag }\end{array}$ & 58 & 332 \\
\hline Hnf6 & $\begin{array}{l}\text { gcaatggaagtaattcagggcag } \\
\text { cgtgacagtcgttgaagaagtac }\end{array}$ & 60 & 471 \\
\hline PDX-1 & $\begin{array}{l}\text { ctttcccgtggatgaaatcc } \\
\text { gtcaagttcaacatcactgcc }\end{array}$ & 60 & 205 \\
\hline
\end{tabular}

\subsection{Western Blot Analysis}

Cells were lysed in buffer containing $50 \mathrm{mM}$ Tris, $150 \mathrm{mM} \mathrm{NaCl}, 1 \%$ Nonidet P40 (Roche; Indianapolis, IN), $0.5 \%$ deoxycholate, $1 \mathrm{~mL}$ protease inhibitor cocktail for every $100 \mathrm{~mL}(5 \mu \mathrm{g} / \mathrm{mL}$ aprotinin, leupeptin, pepstatin, and soybean trypsin inhibitor), and $1 \mathrm{~mL}$ phosphatase inhibitor for every $100 \mathrm{~mL}$. Lysates were clarified by centrifugation at $14,000 \mathrm{~g} \times 2 \mathrm{~min}$ and stored at $-20^{\circ} \mathrm{C}$. Protein concentrations were determined by BioRad protein assay. Total cellular proteins (50 $\mu \mathrm{g} / \mathrm{lane})$ were dissolved by SDS-PAGE, transferred to nitrocellulose membrane incubated with blocking buffer (5\% nonfat dry milk in $1 \times$ Tris Buffered Saline with $0.05 \%$ Tween 20, pH 7.5), and probed with primary antibodies. After incubation with secondary antibodies, peroxidase activity was detected by enhanced chemiluminescence. Densitometric signals from Western blots were analyzed with NIH-ImageJ software (http://rsb.info.nih.gov/ij/) [31]. Protein levels were calculated in arbitrary units (AU) normalized with $\beta$-actin protein levels.

\subsection{Insulin Assay}

Insulin secretion was measured using an enzyme linked immunosorbent assay (ELISA). Cells were cultured in either a high or low glucose environment $(5 \mathrm{mM}$ and $25 \mathrm{mM}$ respectively) for either a 4 or 48 hour duration. Supernatant was collected from culture dishes and placed at $-20^{\circ} \mathrm{C}$ storage. The samples were allowed only 1 freeze-thaw before analysis. The samples were analyzed according to the manufacturer's protocol (Alpco Diagnostics, Salem, NH) by the UNC Center for Gastrointestinal Biology and Disease (CGIBD) Immunotechnology core facility funded by NIH grant\# P30-DK34987and the insulin concentrations calculated using standard curves generated from each assay.

\subsection{Cell Proliferation Assay}

Cell growth and proliferation were measured using the CyQUANT $^{\circledR}$ NF Cell Proliferation Assay Kit (Invitrogen Molecular Probes ${ }^{\text {TM }}$; Eugene, OR) which measures DNA content. Cells were allowed to proliferate in 96 well culture dishes for set time points of $0,1,4,7,14$, and 21 days. On test day growth medium was removed and $50 \mu \mathrm{l}$ of $1 \times$ binding dye from kit added. Cells were further incubated for 30 minutes and then assayed on a BioTek Microplate reader at emission wavelengths of 485 and 530 $\mathrm{nm}$. Absorbance values obtained were then correlated to cell numbers using a standard curve. Standard curve was created previously with a rapidly proliferating cell line.

\section{RESULTS}

Sca-1 is a marker demonstrated on select stem cell po- 
pulations including hematopoietic stem cells, endothelial progenitor cells, adipose stem cells and hepatic stem cells [32-35]. Pancreatic tissue isolated from newborn and adult mice, (12 days and 6 weeks old, respectively) was evaluated for the presence of Sca- $1^{+}$cells. In newborn mice the Sca- $1^{+}$cells are localized in the periductal region (Figure 1(a)). In adult mice Sca- $1^{+}$cells are located in a periductal and peri-islet distribution (Figure 1(b)) with a noted absence of Sca- $1^{+}$cells within the islet matrix.

Pancreatic tissue was subsequently disbursed into a cellular suspension using a combination of mechanical and enzymatic separation techniques followed by enrichment for the Sca- $1^{+}$cells (Sca-PPC) with an established magnetic separation technique. This isolation technique generates an average of $1 \times 10^{6} \mathrm{Sca}-1^{+}$cells from the pancreas and $510^{6} \mathrm{Sca}^{-} 1^{-}$cells. Cellular enrichment for the Sca- $1^{+}$cells was confirmed by flow cytometric analysis (Figure S1-Supplemental Data).

\subsection{Cellular Proliferation and Characterization}

After isolation, the Sca-PPC were placed on tissue culture dishes using a modification of Ta's media conditions [36]. Small colonies of tightly packed cells with large nuclei are seen on the dishes within the first three days of culture (Figure 2a). These colonies rapidly expand in culture forming large colonies. As these cells proliferate they morphologically change with an increase in cytoplasm to nucleus ratio (Figure 2b). Assessing the rate of cellular proliferation using a mitochondrial assay demonstrates an initial slow proliferation rate of the Sca-PPC during the first week of culture followed by an acceleration over the subsequent 14 days in culture (Figure 2c). Due to the periductal location of the Sca-PPC in the pancreatic parenchyma we analyzed the tissue for CK19 expression, a marker associated with ductal cells (e.g. bile ducts, pancreatic ducts, etc.). (Supplemental Figure S2) Immunohistochemistry of the pancreatic tissue demonstrates similar staining patterns for CK19 and Sca-1. We subsequently evaluated the proliferating Sca-PPC for CK19 expression. Figure 3 demonstrates CK19 expression on a day 7 colony of proliferating Sca-PPC. During the in vitro culture period, the Sca-PPC were analyzed for persistence of Sca-1 expression. Figure 4 is a day 3 colony demonstrating persistent Sca-expression on the cells. The nuclei of these cells are counterstained with DAPI.

\subsection{Transcription Factors and Differentiation}

Transcription factor expression associated with pancreatic development and/or mature pancreatic cells was evaluated in the Sca-PPC population. We selected defined time points to evaluate cellular differentiation: immediately after isolation (P0) and in culture after the cells had been passaged (P2 and P4). Murine islets were used as a control for these experiments. The Sca-PPC demonstrates expression of $\mathrm{Hnf6}$ at isolation and at later passage, P4. These cells also expressed Pax6, Nkx2.2, Pdx-1, Ptf1- $\alpha$, Ngn3, NeuroD, nestin, CK19, Insulin 1 and 2 but did not express Glut 2. Figure 5 is a representative set of images from these experiments. Experiments were performed a minimum of three times to confirm the presence or absence of a transcription factor.

Based on the PCR results we assessed Sca-PPC differentiation by comparing markers associated with the endocrine component of the islet and the acinar fraction of the pancreas. Western blot analyses demonstrate de novo low expression levels of glucagon and Ngn3 which persist during cell passage. Insulin receptor- $\alpha$ expression is higher at all three time points in culture, but there are no statistically significant differences in this experiment (Figure 6). The Sca-PPC also demonstrates de novo expression of amylase with persistent expression during cell culture.

$\mathrm{Pdx}-1$ is selectively expressed in $\beta$-cells and is essential for maintenance of phenotype in adult $\beta$-cells [37]. Based on the finding of Pdx-1 by PCR (Figure 5) we performed immunofluorescense analysis of the Sca-PPC colonies to assess cellular expression. Figure 7 demonstrates strong Pdx-1 expression on the majority of colony-forming ScaPPC.

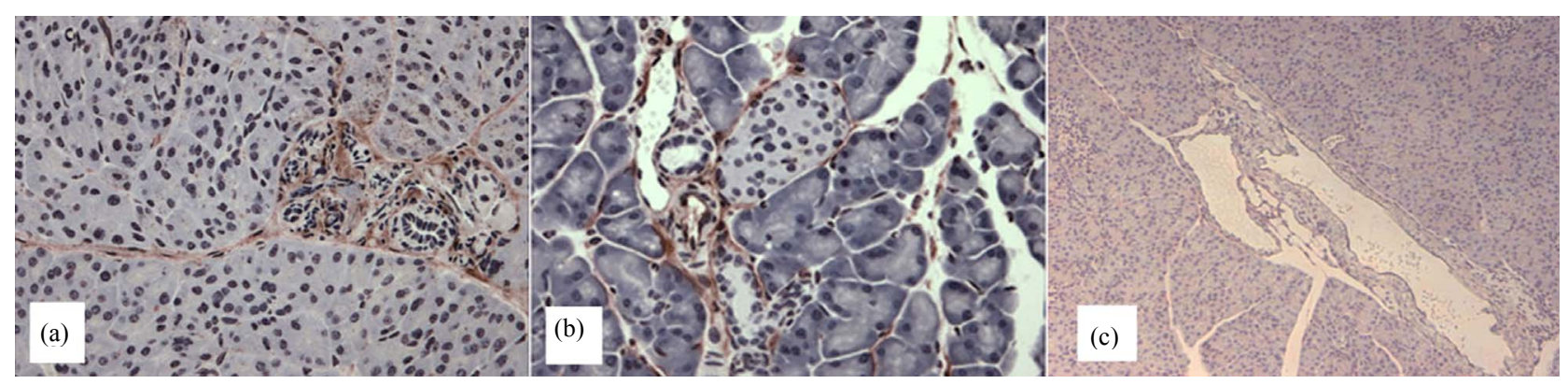

Figure 1. Sca-1 expression in normal murine pancreas tissue; (a) Immunohistochemical staining shows Sca-1 expression along the ductal network of neonate mice 2 weeks of age; (b) Expression is also seen along the ductal epithelium as well as in the peri-islet space of adult mice 6weeks of age; Image (c) represents an isotype control for Sca-1. (a) 20×, (b, c) 10×. 

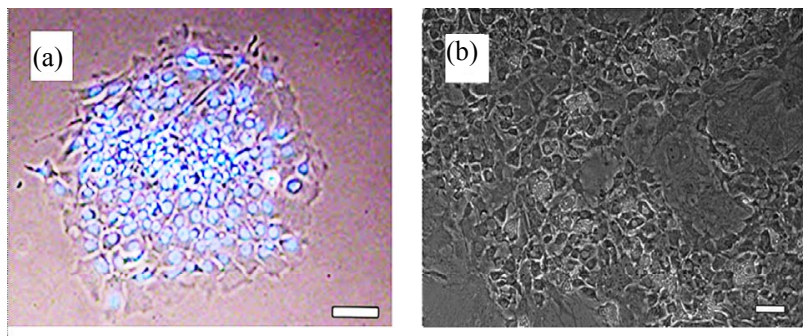

(c)

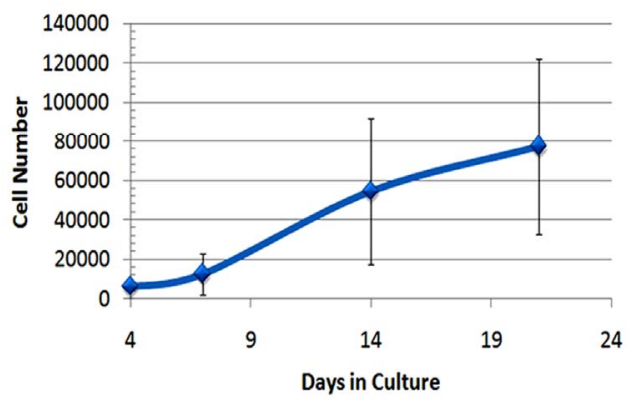

Figure 2. Changes in Sca- $1^{+}$colony morphology throughhout culture. (a) Sca- $1^{+}$colony morphology changes over the duration of standard culture. Initial colonies are similar those seen with other stem cell sources. Colonies are tightly packed with individual small cells each containing a high nuclear to cytoplasm ratio. Image of day 3 colony. Scale bar $20 \mu \mathrm{m}$; (b) By day 21 of culture colonies spread over $90 \%$ of dishes and have a cobble stone type appearance. Scale bar $50 \mu \mathrm{m}$; (c) Proliferation assay to evaluate doubling time of Sca-PPCs. The proliferation is most accentuated between days $7-21$.

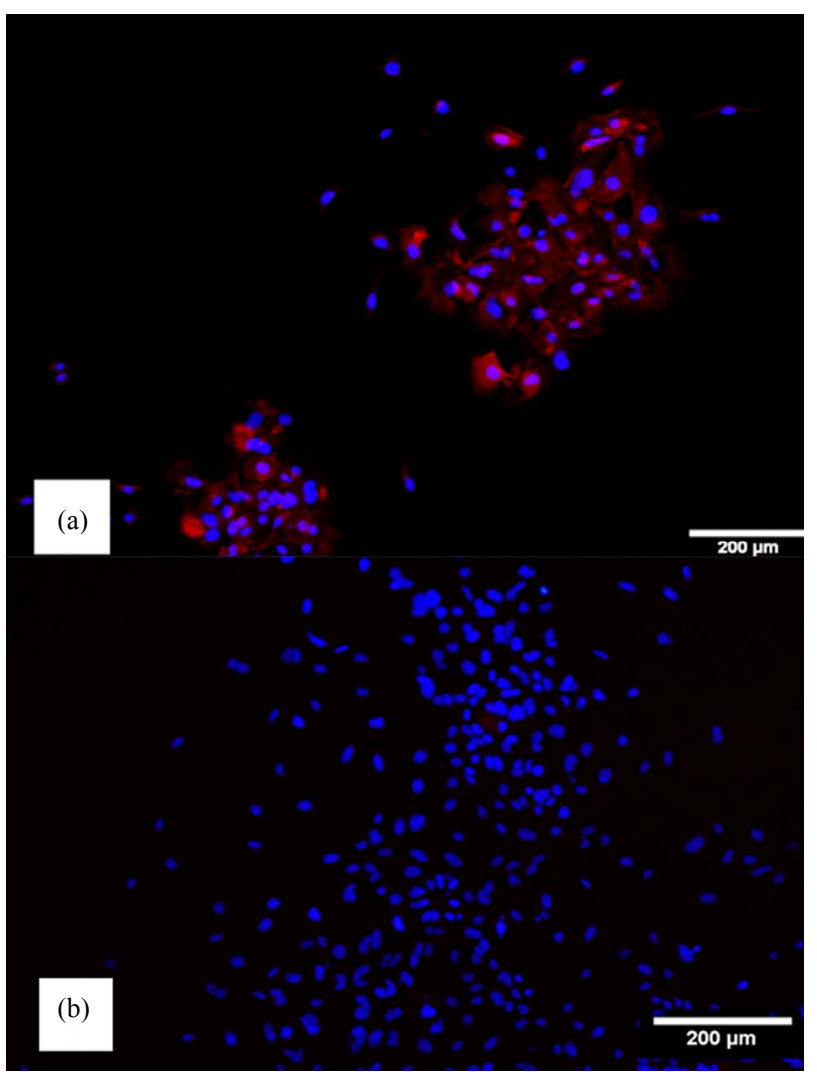

Figure 3. (a) CK19 expression of a day 7 colony. Red correlates with CK19, blue correlates with DAPI staining of nuclei; (b) Corresponding iso-type control. Both images $10 \times$.
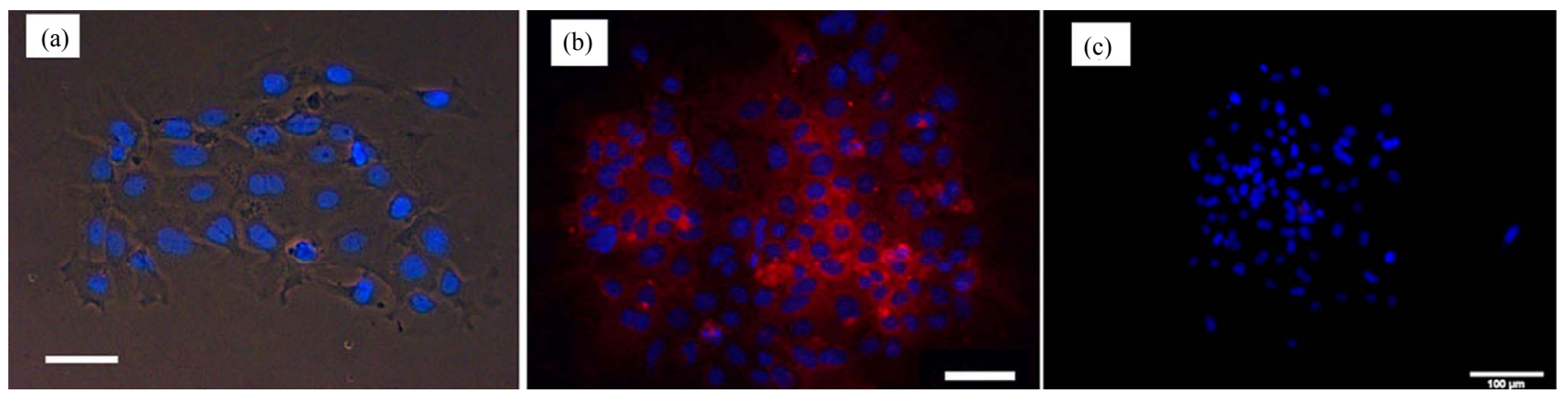

Figure 4. Sca-1 Immunohistochemistry in culture. Colonies in standard culture are formed from Sca- $1^{+}$cells, not contaminating cell types. (a) DAPI staining delineates nuclei within a transmission image of a day 3 colony; (b) Merged DAPI and Sca-1 staining.(c) Isotype control. (a ,b) $20 \times$, scale bar $20 \mu \mathrm{m}$. (c) $10 \times$.

\subsection{Insulin Production of PPC}

Experiments were established to analyze Sca-PPC insulin production looking at both sustained production and rapid response to environmental changes in glucose concentration. The Sca-PPCs demonstrate a basal production of insulin using either low or high glucose concentrations ( $\mathrm{P} 0$ cells) while the cells that have been passaged twice (P2) demonstrate an increase in insulin secretion at both low and high glucose concentrations (Figure 8(a)). In the later passage (P4), insulin production is sim- ilar to the pattern demonstrated when the cells are initially isolated. These assays are based on a 48 hour collection sample and potentially mask rapid changes in insulin secretion. We established a rapid response assay with the Sca-PPC at both low and high glucose concentrations and compared them with freshly isolated islets. Samples were assessed immediately after the media change (30 minutes) and several times over a four hour period. This experiment demonstrates persistent basal insulin production by the Sca-PPC without acute responsiveness to 
changes in glucose concentrations within the media (Figure 8(b)). Sca-PPC insulin production is distinctly different from islets cultured in high glucose concentration media. Comparatively the Sca-PPC have a higher production of insulin in low glucose concentration media experiments compared with islets at the same time points, 30 and 60 minutes respectively (Figure 8b).

\section{DISCUSSION}

Diabetes is currently the seventh leading cause of death, the leading cause of kidney failure, lower limb amputations, and blindness, as well as a major contributor to
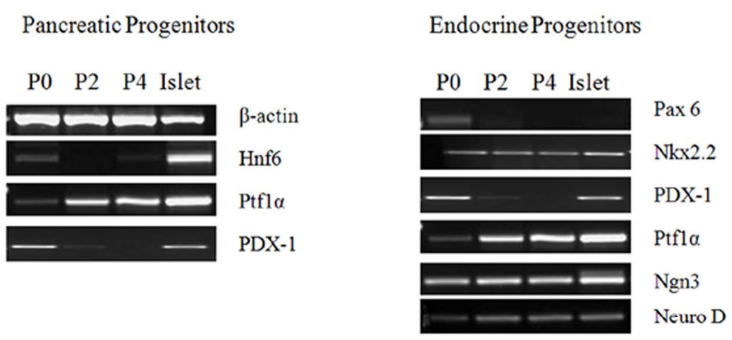

Mature Endocrine Cell
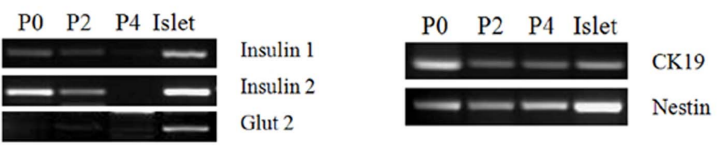

Figure 5. RT-PCR analysis of $\mathrm{Sca}-1^{+}$PPCs. PCR analysis shows that PPCs differentiate toward multiple lineages of pancreatic fate and not toward simply endocrine $\beta$-cell fate. Cells were assayed at passage 0,2 , and 4 for the listed pancreatic transcription factors. Ck19 was also analyzed since its expression was seen in staining in vitro (refer to Figure 3).

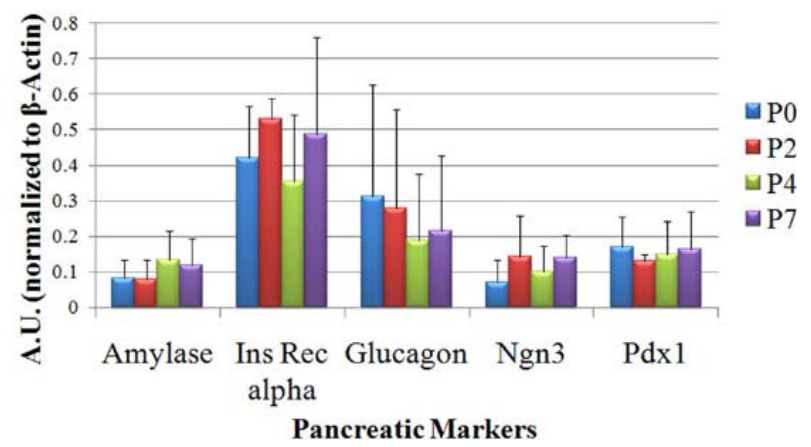

Figure 6. Protein expression of Sca- $1^{+}$PPCs. Western blot analyses performed at passage numbers $0,2,4$, and 7 for pancreatic proteins Amylase, Insulin Receptor Alpha, Glucagon, Ngn3, and Pdx-1. Values are reported in arbitrary units. Results correspond with transcription data in that there is no clear line of differentiation. No statistical significance was observed. Error bars reflect standard error. Arbitrary units are normalized for expression of control protein $\beta$-actin.

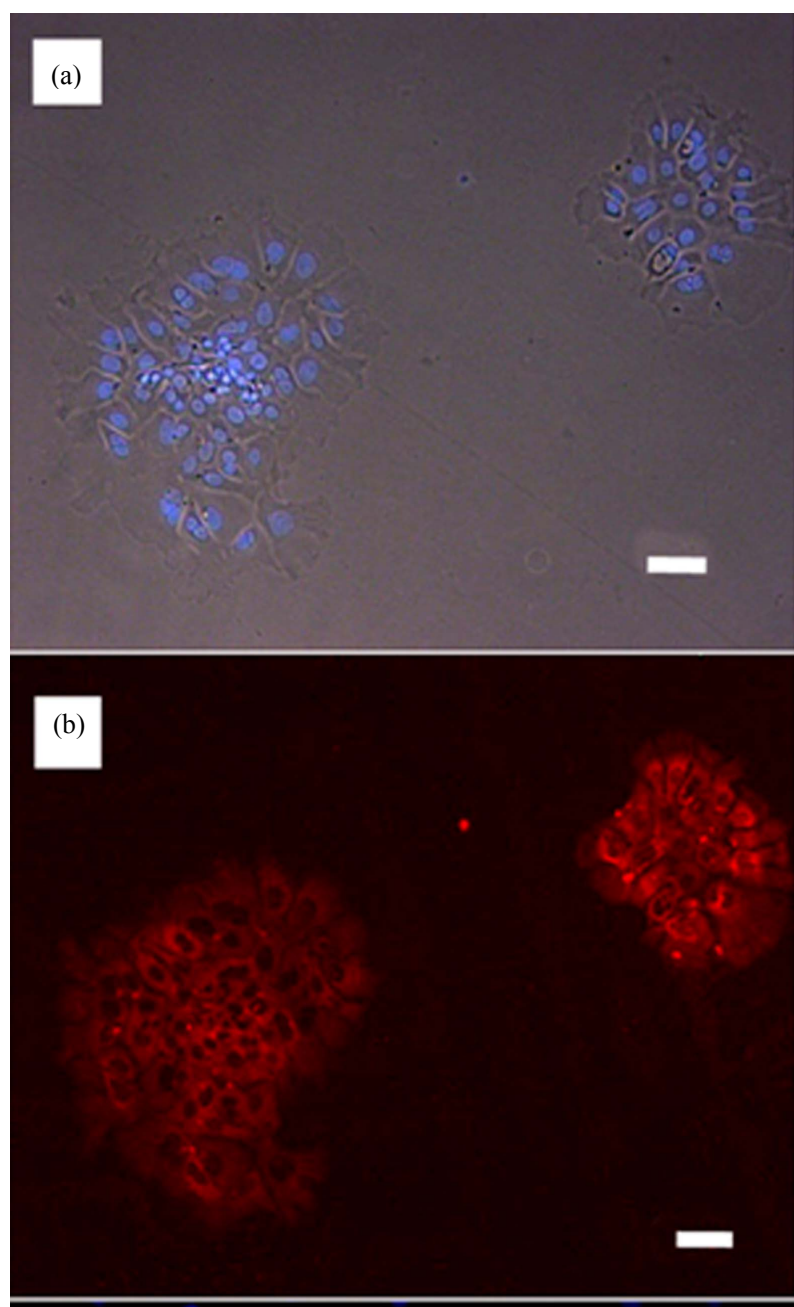

(c)

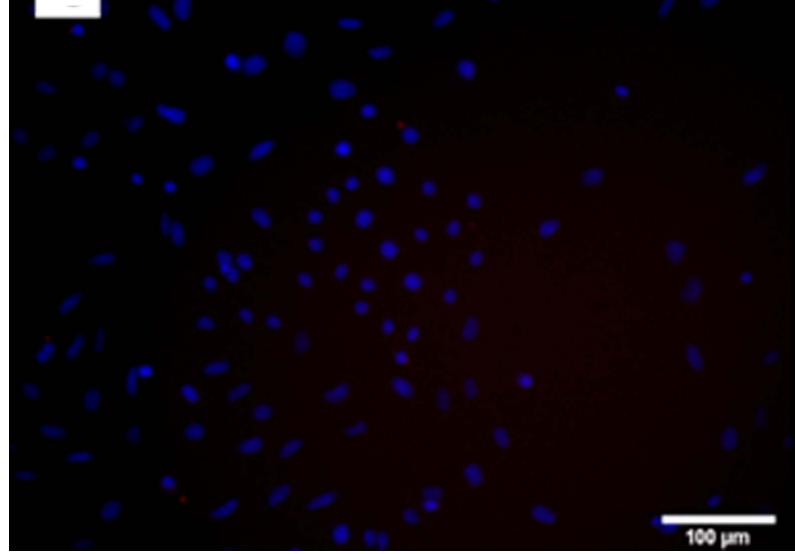

Figure 7. PDX-1 is expressed by all $\beta$-cells of the pancreas and is necessary in formation of $\beta$-cell tissue from progenitor cell origins. PDX-1 is expressed on day 3 Sca- $1^{+}$colonies. Expression is strongly shown in the cytoplasm and not the nuclear region of the cells. (a) Transmission image with DAPI delineating nuclei. (b) PDX-1 expression of the same colonies using PE conjugated antibody. (c) Isotype control. All images 10×. Scale bar for (a) and (B) equals $20 \mu \mathrm{m}$. 


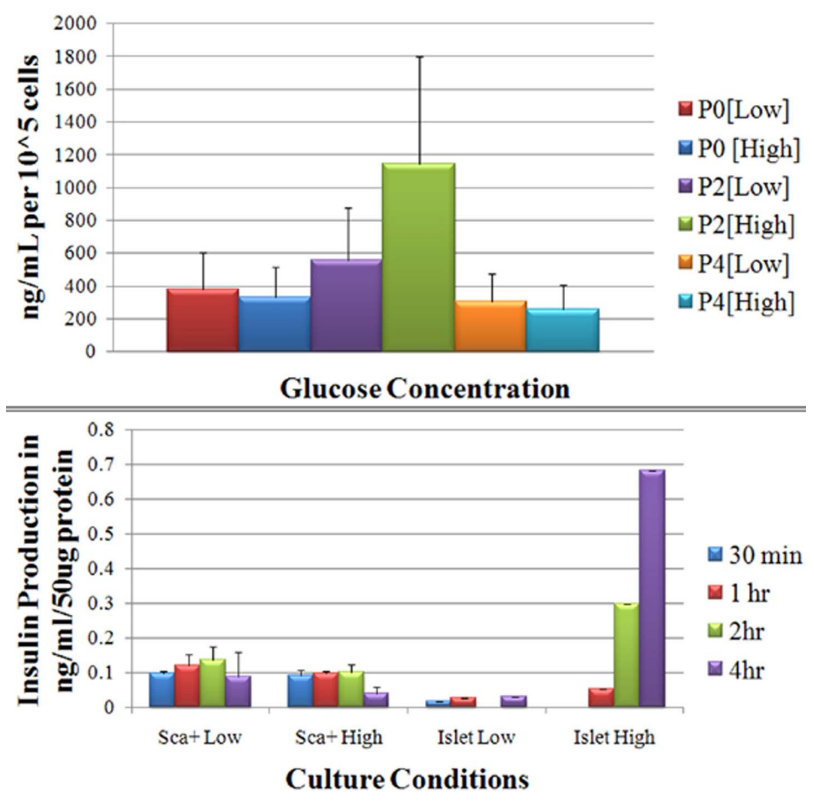

Figure 8. Functional ability of Sca- $1^{+}$PPCs to produce insulin. (Top panel) ELISA was utilized to measure sustained secretion of insulin by neonate ( 2 week) Sca- $1^{+}$donor cells. Cells were stimulated with low $(2.8 \mathrm{mM})$ and high glucose $(40 \mathrm{mM})$ conditions for a 48 hour period and supernatant collected at the conclusion. (Bottom panel) Insulin ELISA was also utilized to measure rapid response to increased glucose conditions. Neonatal cells were stimulated for a 4 hour duration with either high $(40 \mathrm{mM})$ or low $(5.55$ $\mathrm{mM}$ ) glucose conditions in the absence of FBS. Supernatants were collected at $30 \mathrm{~min}, 1,2$, and 4 hours. Cells were assayed at Passage 0 of culture during the time period when small colonies are visible. Mature islets were used as controls. Units are expressed in $\mathrm{ng} / \mathrm{ml}$ normalized for $50 \mu \mathrm{g}$ of protein. No statistical significance was observed. No statistical significance was observed. Error bars represent standard error.

heart disease and stroke in the US [38]. Current treatment options for Type 1 diabetics do not offer physiologically-responsive strict control of blood sugar levels to prevent the development of the previously mentioned complications. Islet cell transplant is a clinically proven method to treat Type I diabetes but it is challenged by severe limitations in donor availability, poor long term success rate, and the requirement for immunosuppression. For these reasons an alternative cell source that could provide glucose responsiveness offers a potential cure to the approximately 2 million newly diagnosed T1D patients each year in the US and the T2D insulin dependent patients [38].

Ongoing debate in the field of stem cell biology questions the existence of a progenitor cell in adult pancreatic tissue. Initial studies have shown that new $\beta$-cells arise as a result of division of pre-existing $\beta$-cells throughout life and after pancreas injury, thus casting doubt on the idea of stem cell contributions $[9,10,39,40]$. This contrasts with others who have shown that a stem cell does exist in the pancreas. Seaberg et al. isolated pancreatic precursors from adult mouse pancreas and demonstrated that the cells are positive for many $\beta$-cell markers and capable of releasing insulin in response to glucose stimulation [28]. However, the amount of insulin produced by these cells was much lower than what is produced by a naturally occurring islet-derived $\beta$-cell. It has also been shown that endocrine precursors can be derived from surgically resected portions of human pancreas and that islets themselves contain a population of mesenchymal stem cells [41,42]. Recently published data bolsters the idea of a precursor cell in pancreas citing the existence of insulin positive multipotent stem cells that give rise to pancreatic and neural lineages and that ameliorate diabetes in animal models [43].

Novel cell surface antibodies are being developed that allow the selective isolation of individual cell populations, (e.g. exocrine cells) providing a useful tool to study adult derived progenitors [44]. Our current study demonstrates the presence of a tissue derived progenitor cell from murine pancreas that is highly proliferative and able to be purified using a select cell surface marker, Sca- $1^{+}$. Other reports demonstrate Sca- 1 expression in progenitor cells of the kidney, liver, cardiac and skeletal muscle, and mammary tissue but to our knowledge this is the first report of a Sca- $1^{+}$progenitor cell residing in murine pancreas [34,45-48].

CK19 is another marker found on the Sca-PPCs during in vivo and in vitro assessment. CK19 is a ductal marker and its expression in murine pancreas is found to overlap with ductal areas positive for Sca-1. In early cell culture experiments all of the Sca-PPC colonies are positive for CK19. This expression of CK19 is important in translating our research to other species. Sca-1 is an antigen that is specific to murine tissues thus an alternative marker is needed when we analyze human tissue. Several groups have reported the possibility of multipotent stem cells associated with ductal areas and the biliary ducts that traverse the pancreas, $[16,49,50]$. The presence of CK19 in our Sca-PPCs may indicate overlap with other isolated precursor cells and offer an opportunity for exploration of new antigens of interest.

Analysis of cultured Sca-PPCs demonstrates variability in the expression of differentiation markers. The cells initially express many of the developmental markers associated with pancreatic and endocrine progenitor cells, including Hnf-6, Ptf1 $\alpha$, PDX-1, Pax6, Nkx2.2, Ngn3, Neu$\mathrm{roD}$, and Nestin. Variability in transcription factor expression is demonstrated throughout the periods of culture that were studied. After initially expressing multiple endocrine developmental markers the Sca-PPCs lose expression of Hnf6 and Pax6 while maintaining expression of Nkx2.2, Ngn3 and Neuro D. Cells also express differ- 
entiated cell markers including Insulin1, Insulin2, Glut2, and CK19. These markers are initially present but by the time the cells have undergone multiple passages the cells lose expression of both Insulin 1 and 2. A possible explanation for the variability of expression could relate to cell-cell interactions in the in vitro cellular environment and the impact on the cells, as cells cultured on a rigid surface have been previously associated with cellular differentiation [51,52]. Maintaining the cells in a 2-D environment thereby limits their ability to develop normally and forces the cells to differentiate based on constraints of the culture vessel as well as causing cell death due to limited space.

PDX-1 is a transcription factor that is present during early development of the pancreas and is essential for the differentiation process into mature $\beta$-cells. For this reason its presence would theoretically be essential in any cell population which has the potential of becoming a functional islet-like cell. Sca-PPCs express PDX-1 throughout culture at the transcriptional level although in vitro analysis demonstrates its expression in the cells' cytoplasm. This is distinct from the typical nuclear location of PDX1. This cytoplasmic localization in the PPCs suggests signal transcription but not translation of the protein into an active form. This observation has been demonstrated by other investigators where PDX-1 is found in an inactive form in the cytoplasm of the islet when glucose concentrations are low. PDX-1 is then activated by phosphorylation when glucose concentrations increase and the active form is translocated to the cell nucleus [53-56].

Protein analysis of the Sca-PPC population shows that cells express the developmental markers Ngn3 and PDX-1 throughout the analyzed periods of culture. The cells also express mature endocrine proteins glucagon and Insulin Receptor $\alpha$. Likewise, the mature exocrine cell marker amylase is expressed throughout the in vitro culture periods. The expression levels of each protein vary throughout the experiment showing the cells' ability for endocrine and exocrine functions.

Sca-PPCs have the functional ability to produce large in vitro amounts of insulin. This is a distinct finding compared to published studies where insulin secretion is very limited in progenitor cell populations. Sca-PPCs from adult mice produce a steady state of insulin across passages and over a 2 day period of culture yet the release is independent of changes in glucose concentration. Neonatal mice are capable of responding to glucose concentration changes at passage 2 , but do not maintain this ability in extended culture. In acute phase experiments there is no increase in insulin secretion from the Sca-PPCs in high glucose conditions over a four hour period. In fact, cells exposed to low glucose conditions released more insulin than their high glucose counterparts. This was in stark contrast to islets which show a drastic increase in insulin secretion at high concentrations and only a minor increase in low glucose conditions. Finding a way to direct the secretion of insulin in response to glucose stimulation in the Sca-PPC's may show that they have potential as a stem cell source capable of treating diabetes.

This study demonstrates the presence of a Sca- $1^{+}$progenitor cell subset in the murine pancreas. The expression of CK19 offers a potential complementary marker for selection of a correlating population in human pancreatic tissue. This co-expression of ductal markers may also offer insight to the origin of these progenitor cells. Based upon the common embryologic development of pancreas and liver from the foregut endoderm the complex ductal networks of both organs likely share a common origin. This is corroborated by the fact that our lab previously identified a Sca- $1^{+}$cell residing in adult murine liver [34]. Regulation of the proliferative and differentiation features of this cell population will be critical as we continue to investigate alternative strategies for glucose regulation in our diabetic population.

\section{REFERENCES}

[1] Engelgau, M.M., et al. (2004) The evolving diabetes burden in the United States. Annals of Internal Medicine, 140, 945-950.

[2] Diabetes Prevention Program Research Group (2009) 10 year follow-up of diabetes incidence and weight loss in the Diabetes Prevention Program Outcomes Study. The Lancet, 374, 1677-1686. doi:10.1016/S0140-6736(09)61457-4

[3] Bonner-Weir, S. and Sharma, A. (2002) Pancreatic stem cells. Journal of Pathology, 197, 519-526. doi:10.1002/path.1158

[4] Teitelman, G., et al. (1993) Precursor cells of mouse endocrine pancreas coexpress insulin, glucagon and the neuronal proteins tyrosine hydroxylase and neuropeptide Y, but not pancreatic polypeptide. Development, 118, 1031-1039.

[5] Zulewski, H., et al. (2001) Multipotential nestin-positive stem cells isolated from adult pancreatic islets differentiate ex vivo into pancreatic endocrine, exocrine, and hepatic phenotypes. Diabetes, 20, 521-533. doi:10.2337/diabetes.50.3.521

[6] Schwitzgebel, V.M., et al. (2000) Expression of neurogenin3 reveals an islet cell precursor population in the pancreas. Development, 127, 3533-3542.

[7] Rovira, M., et al. (2009) Isolation and characterization of centroacinar/terminal ductal progenitor cells in adult mouse pancreas. Proceedings of the National Academy of Sciences of the USA, 107, 75-80. doi:10.1073/pnas.0912589107

[8] Dor, Y. and Melton, D.A. (2008) Facultative endocrine progenitor cells in the adult pancreas. Cell, 132, 183-184. doi:10.1016/j.cell.2008.01.004

[9] Brennand, K., Huangfu, D. and Melton, D. (2007) All beta cells contribute equally to islet growth and mainte- 
nance. PLoS Biology, 5, e163. doi:10.1371/journal.pbio.0050163

[10] Dor, Y., et al. (2004) Adult pancreatic beta-cells are formed by self-duplication rather than stem-cell differentiation. Nature, 429, 41-46. doi:10.1038/nature02520

[11] Liu, H., et al. (2010) Precursor cells in mouse islets generate new \{beta\}-cells in vivo during aging and after islet injury. Endocrinology, 151, 520-528. doi:10.1210/en.2009-0992

[12] Noguchi, H., et al. (2009) Establishment of mouse pancreatic stem cell line. Cell Transplant, 18, 563-571.

[13] Cornelius, J.G., et al. (1997) In vitro generation of islets in long-term cultures of pluripotent stem cells from adult mouse pancreas. Hormone \& Metabolic Research, 29, 271-277. doi:10.1055/s-2007-979036

[14] Ramiya, V.K., et al. (2000) Reversal of insulin-dependent diabetes using islets generated in vitro from pancreatic stem cells. Nature Medicine, 6, 278-282. doi:10.1038/73128

[15] Bonner-Weir, S., et al. (2000) In vitro cultivation of human islets from expanded ductal tissue. Proceedings of the National Academy of Sciences of the USA, 97, 79998004. doi:10.1073/pnas.97.14.7999

[16] Li, W.C., et al. (2010) Activation of pancreaticduct derived progenitor cells during pancreas regeneration in adult rats. Journal of Cell Science, 123, 2792-2802. doi: $10.1242 /$ jcs. 065268

[17] Petropavlovskaia, M. and Rosenberg L. (2002) Identification and characterization of small cells in the adult pancreas: Potential progenitor cells? Cell and Tissue Research, 310, 51-58. doi:10.1007/s00441-002-0614-Z

[18] Kroon, E., et al. (2008) Pancreatic endoderm derived from human embryonic stem cells generates glucoseresponsive insulin-secreting cells in vivo. National Biotechnology, 26, 443-452. doi:10.1038/nbt1393

[19] D'Amour, K.A., et al. (2006) Production of pancreatic hormone-expressing endocrine cells from human embryonic stem cells. National Biotechnology, 24, 13921401.

[20] Kahan, B.W., et al. (2003) Pancreatic precursors and differentiated islet cell types from murine embryonic stem cells: An in vitro model to study islet differentiation. Diabetes, 52, 2016-2024. doi:10.2337/diabetes.52.8.2016

[21] Ku, H.T., et al. (2004) Committing embryonic stem cells to early endocrine pancreas in vitro. Stem Cells, 22, 1205-1217. doi:10.1634/stemcells.2004-0027

[22] Gu, G., Dubauskaite, J. and Melton, D.A. (2002) Direct evidence for the pancreatic lineage: NGN3+ cells are islet progenitors and are distinct from duct progenitors. Development, 129, 2447-2457.

[23] Apelqvist, A., et al. (1999) Notch signalling controls pancreatic cell differentiation. Nature, 400, 877-881. doi: $10.1038 / 21913$

[24] Jensen, J., et al. (2000) Control of endodermal endocrine development by Hes-1. Nature Genetics, 24, 36-44. doi:10.1038/71657

[25] Desgraz, R. and Herrera, P.L. (2009) Pancreatic neurogenin 3-expressing cells are unipotent islet precursors. Development, 136, 3567-3574. doi:10.1242/dev.039214

[26] Yao, Z.X., et al. (2004) In vitro cultivation of human fetal pancreatic ductal stem cells and their differentiation into insulin-producing cells. World Journal of Gastroen- terology, 10, 1452-1456.

[27] Suzuki, A., Nakauchi, H. and Taniguchi, H. (2004) Prospective isolation of multipotent pancreatic progenitors using flow-cytometric cell sorting. Diabetes, 53, 21432152. doi:10.2337/diabetes.53.8.2143

[28] Seaberg, R.M., et al. (2004) Clonal identification of multipotent precursors from adult mouse pancreas that generate neural and pancreatic lineages. Nature Biotechnology, 22, 1115-1124. doi:10.1038/nbt1004

[29] Thorel, F., et al. (2010) Conversion of adult pancreatic alpha-cells to beta-cells after extreme beta-cell loss. $\mathrm{Na}$ ture, 464, 1149-1154. doi:10.1038/nature08894

[30] Strobel, O., et al. (2010) Pancreatic duct glands are distinct ductal compartments that react to chronic injury and mediate shh-Induced metaplasia. Gastroenterology, 138, 1166-1177. doi:10.1053/j.gastro.2009.12.005

[31] Abramoff, M.D., Magelhaes, P.J. and Ram, S.J. (2004) Image processing with image. Journal of Biophotonics International, 11, 36-42.

[32] Khoo, C.P., et al. (2009) Characterization of endothelial progenitor cells in the NOD mouse as a source for cell therapies. Diabetes/Metabolism Research and Reviews, 25, 89-93. doi:10.1002/dmrr.898

[33] Chandra, V., et al. (2009) Generation of pancreatic hormone-expressing islet-like cell aggregates from murine adipose tissue-derived stem cells. Stem Cells, 27, 19411953. doi: $10.1002 /$ stem. 117

[34] Wright, N., et al. (2008) Enrichment of a bipotent hepatic progenitor cell from naive adult liver tissue. Biochemical and Biophysical Research Communications, 366, 367372. doi:10.1016/j.bbrc.2007.11.129

[35] Holmes, C. and Stanford, W.L. (2007) Stem cell antigen-1: Expression, function, and enigma. Stem Cells, 25, 1339-1347. doi:10.1634/stemcells.2006-0644

[36] Ta, M., et al. (2006) The defined combination of growth factors controls generation of long-term-replicating islet progenitor-like cells from cultures of adult mouse pancreas. Stem Cells, 24, 1738-1749. doi:10.1634/stemcells.2005-0367

[37] Gu, G., Brown, J.R. and Melton, D.A. (2003) Direct lineage tracing reveals the ontogeny of pancreatic cell fates during mouse embryogenesis. Mechanisms of Development, 120, 35-43. doi:10.1016/S0925-4773(02)00330-1

[38] Centers for Disease Control and Prevention, (2011) National diabetes fact sheet: National estimates and general information on diabetes and prediabetes in the United States. Atlanta, GA.

[39] Nir, T., Melton, D.A. and Dor, Y. (2007) Recovery from diabetes in mice by beta cell regeneration. Journal of Clinical Investigation, 117, 2553-2561. doi:10.1172/JCI32959

[40] Teta, M., et al. (2007) Growth and regeneration of adult beta cells does not involve specialized progenitors. Developmental Cell, 12, 817-826. doi:10.1016/j.devcel.2007.04.011

[41] Shyu, J.F., et al. (2011) Alleviation of hyperglycemia in diabetic rats by intraportal injection of insulin-producing cells generated from surgically resected human pancreatic tissue. Journal Endocrinol, 208, 233-244.

[42] Carlotti, F., et al. (2011) Isolated human islets contain a distinct population of mesenchymal stem cells. Islets, $\mathbf{2}$, 
164-173.

[43] Smukler, S.R., et al. (2011) The adult mouse and human pancreas contain rare multipotent stem cells that express insulin. Stem Cell, 8, 281-293. doi:10.1016/i.stem.2011.01.015

[44] Dorrell, C., et al. (2011) Isolation of mouse pancreatic alpha, beta, duct and acinar populations with cell surface markers. Molecular and Cellular Endocrinology, 399, 144-150.

[45] Dekel, B., et al. (2006) Isolation and characterization of nontubular sca-1+lin-multipotent stem/progenitor cells from adult mouse kidney. Journal of the American Society of Nephrology, 17, 3300-3314. doi:10.1681/ASN.2005020195

[46] Oh, H., et al. (2003) Cardiac progenitor cells from adult myocardium: Homing, differentiation, and fusion after infarction. Proceedings of the National Academy of Sciences of the USA, 100, 12313-12318. doi:10.1073/pnas. 2132126100

[47] Asakura, A., et al. (2002) Myogenic specification of side population cells in skeletal muscle. Journal of Cell Biology, 159, 123-134. doi:10.1083/jcb.200202092

[48] Welm, B.E., et al. (2002) Sca-1 (pos) cells in the mouse mammary gland represent an enriched progenitor cell population. Developmental Biology, 245, 42-56. doi:10.1006/dbio.2002.0625

[49] Cardinale, V., et al. (2010) Multipotent stem cells in the biliary tree. Italian Journal of Anatomy and Embryology, 115, 85-90.

[50] Nagaya, M., et al. (2009) Adult mouse intrahepatic biliary epithelial cells induced in vitro to become insu- lin-producing cells. Journal of Endocrinology, 201, 37 47. doi:10.1677/JOE-08-0482

[51] Darling, E.M. and Athanasiou, K.A. (2005) Retaining zonal chondrocyte phenotype by means of novel growth environments. Tissue Engineering, 11, 395-403. doi:10.1089/ten.2005.11.395

[52] Ayala, P., Lopez, J.I. and Desai, T.A. (2010) Microtopographical cues in $3 \mathrm{D}$ attenuate fibrotic phenotype and extracellular matrix deposition: Implications for tissue regeneration. Tissue Engeneering Part A, 16, 25192527. doi:10.1089/ten.tea.2009.0815

[53] Macfarlane, W.M., et al. (1999) Glucose stimulates translocation of the homeodomain transcription factor PDX1 from the cytoplasm to the nucleus in pancreatic beta-cells. Journal of Biological Chemistry, 274, 1011-1016. doi:10.1074/jbc.274.2.1011

[54] Andrali, S.S., et al. (2008) Glucose regulation of insulin gene expression in pancreatic beta-cells. Biochemical Journal, 415, 1-10. doi:10.1042/BJ20081029

[55] Guillemain, G., et al. (2004) Importin betal mediates the glucose-stimulated nuclear import of pancreatic and duodenal homeobox-1 in pancreatic islet beta-cells (MIN6). Biochemical Journal, 378, 219-227. doi:10.1042/BJ20031549

[56] Rafiq, I., et al. (2000) Glucose-stimulated preproinsulin gene expression and nuclear trans-location of pancreatic duodenum homeobox-1 require activation of phosphatidylinositol 3-kinase but not p38 MAPK/SAPK2. Journal of Biological Chemistry, 275, 15977-15984. doi:10.1074/jbc.275.21.15977

\section{Supplemental Images}

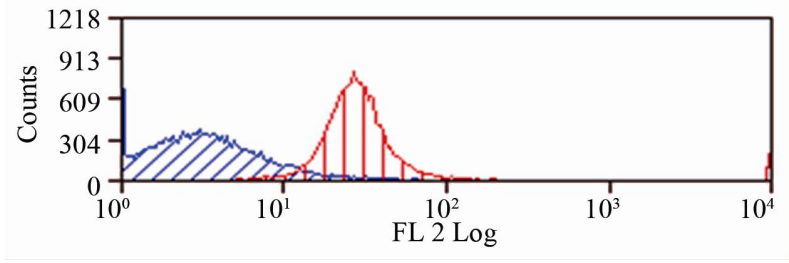

Figure S1. Flow cytometry analysis of immuno-magnetically separated Sca- $1^{+}$PPCs. MACS separation generates a reliable Sca- $1^{+}$cell population. Flow cytometry analysis shows unlabeled cells (blue diagonal hash lines) as a distinct population from PE labeled $\mathrm{Sca}^{+}$cells (red vertical hashed lines), verifying purity in magnetic separation.

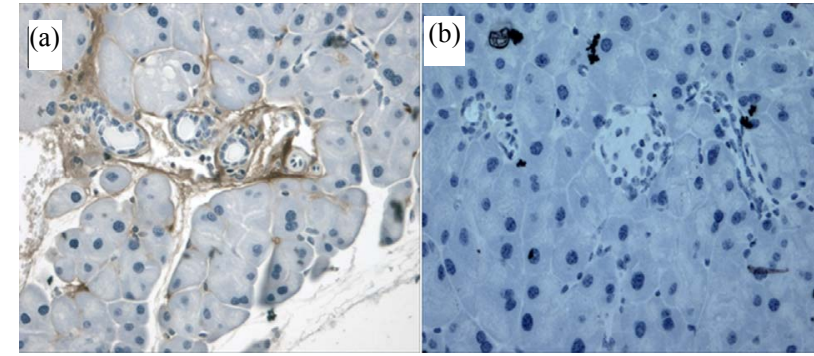

Figure S2. (a) Cytokeratin19 is a common marker of ductal epithelium and serves as a control to delineate the rich ductal network of the pancreas; (b) Corresponding isotype control. Both images $20 \times$. 